\title{
Characteristics of Veterans Health Administration chiropractors and chiropractic clinics
}

\author{
Anthony J. Lisi, DC; ${ }^{1-2 *}$ Christine Goertz, DC, PhD; ${ }^{3}$ Dana J. Lawrence, DC, MMedEd; ${ }^{4}$ Preeti Satyanarayana, \\ MD, MPH $^{5}$ \\ ${ }^{1}$ Veterans Health Administration, Office of Rehabilitation Services, Washington, DC; ${ }^{2}$ Chiropractic Service, Department \\ of Veterans Affairs Connecticut Healthcare System, West Haven, CT; ${ }^{3}$ Research and Health Policy, ${ }^{4}$ Center for Teaching \\ and Learning, and ${ }^{5}$ Palmer Center for Chiropractic Research, Palmer College of Chiropractic, Davenport, IA
}

\begin{abstract}
Chiropractic services have been delivered on station at select Veterans Health Administration (VHA) medical facilities since late 2004. No published data describing the characteristics of VHA chiropractic physicians (chiropractors) and chiropractic clinics exist at a national level. This study was designed to examine elements of the structures of chiropractic services in VHA settings. Web-based survey methods were used to question all chiropractors in VHA facilities $(N=36)$. Data were obtained from 33 providers, yielding a 91.6\% response rate. Most respondents were full-time VHA employees, while others were part-time employees or contractors. Differences were found in prior training, integrated practice, and academic or research experience. Of the respondents, $88 \%$ ranked low back pain as the most common patient complaint seen in practice and $79 \%$ ranked cervical pain the second most common complaint. Of the new patient consultations, $67.6 \%$ originated from primary care, $9.4 \%$ from pain management, and $6.2 \%$ from physiatry. Most respondents were similar in their reported use of diagnostic and therapeutic procedures, but their reported rates of participation in various facility activities were different. Further work is needed for researchers and policy makers to more fully understand the integration and delivery of chiropractic services in VHA settings.
\end{abstract}

Key words: chiropractic clinics, chiropractors, Department of Veterans Affairs, health services, integration, physical medicine, providers, rehabilitation services, survey research, veteran.

\section{INTRODUCTION}

In July 2004, the Veterans Health Administration (VHA) Directive 2004-035 established the provision of chiropractic services on station within VHA [1]. This directive was issued in response to a Congressional mandate (Public Law 107-135), advocated by Veterans Service Organizations and chiropractic professional associations. The directive specifies that chiropractic services are for management of neuromusculoskeletal conditions and are included in the standard Medical Benefits Package for all enrolled veterans. Furthermore, these services are to be delivered on station at a minimum of one facility in each Veterans Integrated Service Network, by full- or part-time hiring or contracting with licensed chiropractic physicians (chiropractors).

Abbreviations: SIIB = Samueli Institute for Information Biology, VA $=$ Department of Veterans Affairs, VHA = Veterans Health Administration.

*Address all correspondence to Anthony J. Lisi, DC; Chiropractic Service, VA Connecticut Healthcare System, 950 Campbell Avenue, Building 2, 4th Floor East, West Haven, CT 06516; 203-932-5711; fax: 203-937-4984.

Email: Anthony.Lisi@va.gov

DOI:10.1682/JRRD.2009.01.0002 
By late 2004, VHA had identified 26 facilities at which to introduce these services and the first chiropractic clinics were established. In fiscal year 2005, more than 4,000 veterans received on-station chiropractic services in VHA. By fiscal year 2008, the program had expanded to 36 clinics and more than 12,000 veterans received services. During this period, the number of clinics increased by 38 percent and the number of veterans seen at these clinics tripled.

To date, no national-level data have been presented regarding the characteristics of VHA chiropractors and related clinical structures. Previous work has described chiropractors and their patients in private practice in the United States and Canada [2-5]. This research has shown significant variation among providers regarding professional training, practice parameters, academic experience, and scholarly activity.

Chiropractors typically manage spinal pain or other musculoskeletal complaints. In private practice settings, the average chiropractic patient is aged 40 to 50 , slightly more than half are female, and less than 10 percent are referred by medical physicians [2]. In VHA, almost half the patient population is aged 60 and only about 7 percent are female [6]. By directive, all patients seen in chiropractic clinics must be referred by medical providers. Thus, VHA chiropractic patients are likely to represent a substantially different demographic than those seen in private chiropractic practice. Moreover, since the introduction of chiropractic services was centrally mandated yet locally implemented, natural variation is expected in clinical structures.

This study describes characteristics of the chiropractors serving in VHA, as well as other elements of chiropractic service delivery in VHA.

\section{METHODS}

\section{Study Design and Participants}

The project was designed as a descriptive observational survey. The study population included all chiropractors in the VHA system, excluding one who is an author of this article. At the beginning of this study, all other chiropractors on record with the VHA Department of Patient Care Services were contacted and invited to participate. Chiropractors who became VHA providers after the study began were not included. The Department of Veterans Affairs (VA) Connecticut Healthcare System and Palmer College of Chiropractic Institutional Review Boards approved the study.

Participants were asked to complete a survey assessing provider demographics, aspects of their training, and clinical practice characteristics. Participants were also asked to provide information about patient demographics, conditions, evaluation and management, and clinical care.

\section{Data Collection and Management}

The survey of provider characteristics is modeled after previous surveys of chiropractors in private practice, notably the work of Nyiendo et al. [7] and Hurwitz et al. [4]. Because of the small number of chiropractors in VHA, we chose not to pilot the survey in our sample to avoid potentially biasing some respondents.

Subjects completed the survey anonymously. Participants were provided access codes to the Web-based survey, and only the Web developer had access to the codes. We analyzed responses using SPSS, version 15 (SPSS Inc; Chicago, Illinois). Descriptive results are reported.

\section{RESULTS}

The survey was completed by 33 of the 36 providers solicited, for a response rate of 91.6 percent. The majority of respondents (94\%) were male, and the mean age was 45.8 years (range $30-68)$. A large percentage (88\%) were non-Hispanic white. Eight respondents (24\%) were veterans themselves.

\section{Employment Characteristics}

Of the respondents, 22 (67\%) were VHA employees, $4(12 \%)$ were without compensation academic-affiliate appointees, 4 (12\%) were contracted providers, and 3 (9\%) were on-station fee-basis consultants. Fifty-eight percent of respondents worked a full 40-hour week at the VHA facility, while thirty-three percent worked part-time, an average of 13.5 hours a week (range 7-39 h).

Twenty-nine (88\%) respondents were based at VHA medical centers, two (6\%) at community-based outpatient clinics, and two (6\%) reported serving both types of facilities. Most respondents (67\%) were the only chiropractors at their facility, whereas others (30\%) reported either one

\footnotetext{
*Full text of survey is available at http://www.palmer.edu/
} research content.aspx?id=7000/. 
or two other chiropractors working at the same facility. Most chiropractic clinics (48\%) are administratively aligned under Physical Medicine and Rehabilitation Service, whereas some $(18 \%)$ were aligned under Medical Service, and few (6\% each) were under Primary Care, Ambulatory Care, and Surgical Services. (Throughout this article, when only top categories are reported, the values do not always equal $100 \%$.) Most respondents provide clinical services without assistants. Only 18 percent reported that nursing staff contributes to patient care in their clinics, and another 18 percent use chiropractic assistants or other clinical assistants.

Thirty-six percent of respondents reported gross annual compensation in the $\$ 80,001$ to $\$ 100,000$ range for chiropractic service delivery in VHA. Twenty-one percent reported a range of $\$ 60,001$ to $\$ 80,000$, and twenty-seven percent reported up to $\$ 60,000$. Six percent reported a range of $\$ 100,001$ to $\$ 120,000$.

\section{Provider Characteristics}

Of the respondents, 12 percent had been in practice for $\leq 5$ years, 36 percent from 6 to 15 years, 36 percent from 16 to 25 years, and 12 percent for $>25$ years. These figures represent total years of practice experience, not just those in VHA settings. Few respondents reported hospital-based training before working at VHA. Thirtythree percent reported some hospital training as a chiropractic student, but just under eighteen percent reported any postgraduate hospital-based training. However, about half the respondents reported prior practice experience in either a hospital or multidisciplinary private practice setting (48\% and 55\%, respectively). Despite this result, before the chiropractors began working at the VHA, the number of cases seen in consultation from medical providers was generally low for most respondents. About 67 percent reported previously seeing from 0 to 6 such consultations a month, and 27 percent reported 6 or more a month.

Most respondents had little experience in scholarly and academic activities. Sixty-seven percent reported no previous authorship of peer-reviewed publications, and thirty percent reported having authored one or more. Only four respondents stated they had been a principal investigator on a funded research project. A minority of subjects reported experience in chiropractic academia. Thirty-six percent reported experience in undergraduate teaching, and twenty-seven percent in postgraduate teaching. However, 24 percent were currently engaged in training chiropractic students and/or other trainees at the VHA facility.

\section{Clinical Practice}

The majority of respondents, 88 percent, ranked low back pain as the most common patient complaint seen in practice. Cervical pain was ranked the second most common compliant by 79 percent of respondents. Across all respondents, the mean percent frequency of conditions seen within the past week is reported as follows:

- Low back complaint: 47.7.

- Neck complaint: 21.4.

- Thoracic complaint: 9.8.

- Lower-limb complaint: 7.4.

- Headache: 6.4.

- Upper-limb complaint: 6.2.

- Wellness: 0.8.

- Visceral complaint: 0.3.

Subjects reported 67.6 percent of their new patient consultations originated from primary care. Additionally, 9.4 percent of consultations came from pain management, 6.2 percent from physiatry, 3.7 percent from orthopedic surgery, and 3.6 percent from neurology. Other consulting services reported include neurosurgery, emergency department, rheumatology, podiatry, and spinal cord injury.

Evaluation and management procedures used directly by chiropractors are listed in Table 1. Most respondents were similar in their use of history and musculoskeletal and/or neurological physical examination procedures.

Chiropractors may be privileged to deliver a number of different therapeutic interventions, including various

Table 1.

Group mean frequency (\%) of evaluation and management procedures used by chiropractors surveyed $(N=33)$.

\begin{tabular}{lrcc}
\hline \multicolumn{1}{c}{ Procedure } & $\begin{array}{c}\text { Often/ } \\
\text { Always }\end{array}$ & Sometimes & $\begin{array}{c}\text { Seldom/ } \\
\text { Never }\end{array}$ \\
\hline Clinical & 100 & 0 & 0 \\
Oral History & 94 & 3 & 3 \\
Orthopedic Testing & 94 & 6 & 0 \\
Soft Tissue Palpation & 91 & 6 & 3 \\
Medical Record Review & 91 & 9 & 0 \\
Range of Motion & 88 & 9 & 3 \\
Joint Palpation & 82 & 15 & 3 \\
Neurological Testing & 79 & 9 & 12 \\
Postural Examination & 36 & 18 & 45 \\
Vital Signs & & & \\
Imaging/Laboratory & 75 & 18 & 3 \\
X-ray & 30 & 55 & 15 \\
Advanced Imaging & 12 & 33 & 54 \\
Laboratory Studies & 12 & 33 & 54 \\
Electrodiagnostic Studies & &
\end{tabular}


methods of spinal manipulation. The most frequent procedure used was the Diversified technique, the manipulative procedure most studied in previous clinical trials and reported to be in highest usage within the chiropractic profession [5,8].

Treatments other than spinal manipulation are listed in Table 2. Most commonly reported was patient education and therapeutic exercise, used often or always by 88 and 76 percent of respondents, respectively.

Patients referred from the chiropractic clinics to other services most commonly were redirected to primary care (34.8\%). Additional services to which consultations were sent were physiatry (9.5\%), pain management $(9.5 \%)$, orthopedic surgery (7.9\%), and neurology (5.3\%). Other services that chiropractors consulted include neurosurgery, rheumatology, physical therapy, occupational therapy, and kinesiotherapy.

\section{Provider Integration}

Participation in integrated activities within the medical center varied among respondents (Table 3). About half the respondents took part in multidisciplinary rounds and inservice presentations. Twenty-one percent reported often or always participating in hospital committee work, but sixty-four percent said they seldom or never did.

\section{DISCUSSION}

This study presents the first description of the chiropractors and clinics comprising the VHA chiropractic program. Because the chiropractic program is a new clinical service for VHA, many questions exist as to the quality, effectiveness, and cost-effectiveness of chiropractic care for patients of VHA. This article provides a preliminary understanding of the introduction of this new service to VHA, and these results can provide a context for follow-up studies that will ultimately assess quality of care.

Our results show a wide variation in provider characteristics and clinical structures. This finding was expected as part of the natural variation in delivering new services. Although the introduction of chiropractic services to VHA was mandated centrally, provider selection and clinical implementation were determined locally.

The variation in provider characteristics is consistent with previous reports of chiropractors in private practice [2-4]. We note that all providers met the minimum VHA Chiropractor Qualification Standards; yet few reported qualifications required for higher grade placement such as
Table 2.

Group mean frequency (\%) of nonmanipulative treatment procedures used by chiropractors surveyed $(N=33)$.

\begin{tabular}{lccc}
\hline \multicolumn{1}{c}{ Procedure } & $\begin{array}{c}\text { Often/ } \\
\text { Always }\end{array}$ & Sometimes & $\begin{array}{c}\text { Seldom/ } \\
\text { Never }\end{array}$ \\
\hline Patient Education & 88 & 9 & 3 \\
Exercise & 76 & 24 & 0 \\
Physical Modalities & 36 & 21 & 42 \\
Acupressure & 30 & 27 & 42 \\
Massage & 27 & 6 & 67 \\
Orthoses & 15 & 48 & 36 \\
Acupuncture & 6 & 0 & 94 \\
Nutritional Supplements & 0 & 12 & 88 \\
Homeopathy & 0 & 3 & 97 \\
\hline \hline
\end{tabular}

Table 3.

Group mean participation (\%) in select facility-integrated activities reported by chiropractors surveyed $(N=33)$.

\begin{tabular}{lccc}
\hline \multicolumn{1}{c}{ Activity } & $\begin{array}{c}\text { Often/ } \\
\text { Always }\end{array}$ & Sometimes & $\begin{array}{c}\text { Seldom/ } \\
\text { Never }\end{array}$ \\
Training Chiropractic & 39 & 3 & 58 \\
$\quad$ Students & & & \\
Multidisciplinary Rounds & 33 & 15 & 48 \\
In-Service Presentations & 27 & 24 & 45 \\
Quality Improvement & 24 & 27 & 45 \\
Hospital Committee Work & 21 & 9 & 64 \\
Research & 18 & 15 & 64 \\
Training Other Trainees & 9 & 9 & 78 \\
\hline \hline
\end{tabular}

academic and research experience, and few reported prior hospital-based training. Historically, chiropractic training has not been integrated with universities and medical centers; thus, the opportunities and infrastructure for chiropractors to be mentored in research and interdisciplinary practice models have been limited.

Directive 2004-035 recommends that facilities privilege chiropractors consistent with the individual's state licensure and clinical competence. We did not assess the variations, if any, in privileging and clinical policies from site to site. We expect that differences in privileging, service agreements, and clinical care pathways do exist; yet, the full extent of these differences would be difficult to capture with closed-ended survey questions. Thus, we plan to measure these differences in follow-up research using stakeholder focus groups and interviews.

While we did not attempt to correlate provider/employment characteristics with clinical/integration characteristics, most likely, the experiences of many of these clinicians will differ. We expect that a chiropractor with prior hospitalbased training, a background in research, and academics on a full-time staff appointment will experience different 
operational integration into a VA facility than a clinician with no prior hospital, research, or academic experience working a 7-hour-a-week contract. However, assessing the optimal provider/employment characteristics was beyond the scope of this article.

Prior reports of chiropractic services in VHA have been presented by one investigator, Andrew Dunn, of the VA Western New York Healthcare System in Buffalo (Dunn et al.) [9]. His initial work analyzed the first 100 chiropractic consultation requests received in the Buffalo chiropractic clinic [9]. Dunn found that most of the requests were for male patients (88\%), with an average age of 55. Half of these patients had a service-connected disability. Most of the consultations were for lumbar spine pain $(n=82)$, with the remaining scattered in the cervical $(n=19)$ and thoracic regions $(n=7)$ and limbs $(n=4)$. Previous treatments included medical management $(n=$ $67)$, physical therapy $(n=49)$, previous chiropractic care $(n=19)$, acupuncture $(n=4)$, surgery $(n=3)$, and massage $(n=2)$. The most common department requesting chiropractic consultation was primary care (58\%), with orthopedics ranked second (22\%).

In a follow-up article, Dunn and Passmore looked at a retrospective review of completed chiropractic consultations in $2006(n=354)$ [10]. Again, this review was limited to the VA Western New York Healthcare System and cannot be generalized beyond that system. They also found that primary care was the main source of patients. The average patient was a 55-year-old male with low back pain. In general, the patient base was overweight and had a 27.43 percent service-connected disability. For those using chiropractic services, the mean number of chiropractor treatments was 6.44 (standard deviation 4.65, 95\% confidence interval 5.9-6.9). Over half the patients received some combination of spinal manipulation with spinal mobilization.

This article is the first to describe the characteristics of VHA chiropractors and clinical structures at a national level. Our results expand on the work just noted to provide a preliminary picture of these providers and clinical services that are a new addition to VHA. Future work is needed to more fully understand the determinants and consequences of variation in chiropractic service implementation. This understanding may then provide a context for assessing issues of quality and efficiency in care delivery.

This study has several limitations. We present descriptive data gathered by provider survey, which is subject to expected limitations associated with survey data, including potential recall or reporting bias by respondents. Our sample size is small $(n=33)$; however, this sample represents a 91.6 percent response rate. These results are limited to VHA chiropractors and chiropractic clinics and cannot be extrapolated to other providers or settings.

\section{CONCLUSIONS}

This study presents the first description of VHA chiropractors and chiropractic clinics. We found a wide variation in provider characteristics, employment arrangements, and facility integration. Conversely, we found much similarity in the reported cases seen and clinical examination and therapeutic procedures used. Further work is needed to more fully understand the implementation and delivery of chiropractic services in VHA settings.

\section{ACKNOWLEDGMENTS}

\section{Author Contributions:}

Study concept and design: A. J. Lisi, C. Goertz, D. J. Lawrence, P. Satyanarayana.

Acquisition of data: D. J. Lawrence, P. Satyanarayana.

Analysis and interpretation of data: A. J. Lisi, C. Goertz, D. J.

Lawrence, P. Satyanarayana.

Drafting of manuscript: A. J. Lisi, C. Goertz, D. J. Lawrence,

P. Satyanarayana.

Obtained funding: C. Goertz.

Financial Disclosures: The authors have declared that no competing interests exist.

Funding/Support: This material was based on work supported in part by the Samueli Institute for Information Biology (SIIB) for the project entitled Association of Chiropractic Colleges-Research Agenda Conference, SIIB Project Number 1 EA-0000056.

Participant Follow-Up: Subjects were VA employees or contractors. These subjects were informed of the pending publication.

\section{REFERENCES}

1. Chiropractic care. Washington (DC): Veterans Health Administration; 2004 Jul 16. Directive 2004-035.

2. Mootz RD, Cherkin DC, Odegard CE, Eisenberg DM, Barassi JP, Deyo RA. Characteristics of chiropractic practitioners, patients, and encounters in Massachusetts and Arizona. J Manipulative Physiol Ther. 2005;28(9):645-53.

[PMID: 16326233]

DOI:10.1016/j.jmpt.2005.09.019

3. Coulter ID, Hurwitz EL, Adams AH, Genovese BJ, Hays R, Shekelle PG. Patients using chiropractors in North 
America: Who are they, and why are they in chiropractic care? Spine. 2002;27(3):291-98. [PMID: 11805694]

DOI:10.1097/00007632-200202010-00018

4. Hurwitz EL, Coulter ID, Adams AH, Genovese BJ, Shekelle PG. Use of chiropractic services from 1985 through 1991 in the United States and Canada. Am J Public Health. 1998;88(5):771-76. [PMID: 9585743]

DOI:10.2105/AJPH.88.5.771

5. Christensen MG, Kerkhoff D, Kollasch MW; National Board of Chiropractic Examiners. Job analysis of chiropractic: A project report, survey analysis, and summary of the practice of chiropractic in the United States. Greeley (CO): National Board of Chiropractic Examiners; 2000. p. 53-62.

6. Office of the Assistant Secretary for Policy and Planning. Veteran population model: VetPop 2004 version 1.0 [Internet]. Washington (DC): Department of Veterans Affairs; 2007. Available from: http://www.nhpco.org/files/public/veterans/ VA\%20101\%20Documents/VetPopModel.pdf/.

7. Nyiendo J, Haas M, Goodwin P. Patient characteristics, practice activities, and one-month outcomes for chronic, recurrent low-back pain treated by chiropractors and family medicine physicians: A practice-based feasibility study. J Manipulative
Physiol Ther. 2000;23(4):239-45. [PMID: 10820296] DOI:10.1016/S0161-4754(00)90170-2

8. Bronfort G, Haas M, Evans R, Kawchuk G, Dagenais S. Evidence-informed management of chronic low back pain with spinal manipulation and mobilization. Spine J. 2008; 8(1):213-25. [PMID: 18164469] DOI:10.1016/j.spinee.2007.10.023

9. Dunn AS, Towle JJ, McBrearty P, Fleeson SM. Chiropractic consultation requests in the Veterans Affairs Health Care System: Demographic characteristics of the initial 100 patients at the Western New York medical center. J Manipulative Physiol Ther. 2006;29(6):448-54. [PMID: 16904490] DOI:10.1016/j.jmpt.2006.06.002

10. Dunn A, Passmore S. Consultation request patterns, patient characteristics, and utilization of services within a Veterans Affairs medical center chiropractic clinic. Mil Med. 2008; 173(6):599-603. [PMID: 18595426]

Submitted for publication January 8, 2009. Accepted in revised form June 17, 2009. 